\title{
Coinfections in Intensive Care Unit with pulmonary tuberculosis and mucormycosis: A clinical dilemma
}

\author{
Pratibha Dube, Richa Saroa, Sanjeev Palta
}

\section{Introduction}

Dual infections in intensive care might be encountered, especially in immunocompromised patients. Most of the dual infections cause a diagnostic dilemma and require a high degree of suspicion in susceptible individuals. However, dual infections with mucormycosis may follow a fulminant course and may be associated with higher mortality and morbidity. ${ }^{[1]}$ We hereby report coinfection of pulmonary tuberculosis (TB) with mucormycosis in a diabetic male that worsened rapidly in spite of the antimicrobial therapy targeted toward the same.

\section{Case Report}

Patient relatives have consented to the publication of the medical records for academic purpose. A 72-year-old nonsmoker male, known case of diabetes mellitus type II since 10 years, on oral hypoglycemic agents, presented with the complaints of altered sensorium with low-grade fever associated with pain abdomen and vomiting and was diagnosed to have severe urinary tract infection. He

From:

Department of Anesthesia and Critical Care, Government Medical College and Hospital, Chandigarh, India

\section{Correspondence:}

Dr. Richa Saroa, Department of Anesthesia and Critical Care, Government Medical College and Hospital, Chandigarh, India.

E-mail: richajayant@ rediffmail.com

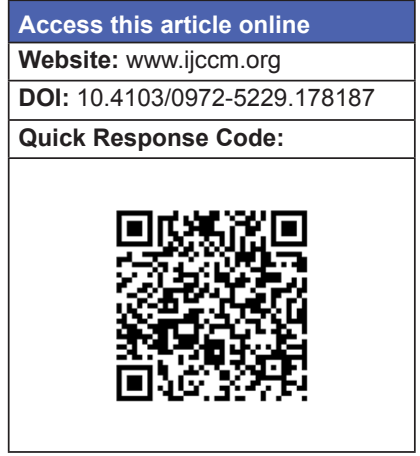

was being managed in the ward for diabetic ketoacidosis for the same and was doing well until the $9^{\text {th }}$ day of admission when he developed cough, expectoration, and difficulty in breathing. Subsequently, the patient was intubated and transferred to Intensive Care Unit (ICU) for mechanical ventilation with Synchronized Intermittent Mandatory Ventilation mode and further managed according to the institutional protocol. All routine biochemical and hematological investigations including the chest X-ray (CXR) were sent. Blood sugar levels were found to be $460 \mathrm{mg} \%$ and total lymphocyte count was $1200 \mathrm{~mm}^{3}$, and urine ketones were found to be positive. Arterial blood gas on the day of admission at $\mathrm{FiO}_{2}$ of 0.6 had $\mathrm{PaO}_{2} / \mathrm{FiO}_{2}$ (PF) ratio of 261 . Empirical antibiotic therapy and vasopressor support with noradrenaline $(0.5 \mu \mathrm{g} / \mathrm{kg} / \mathrm{min})$ was started as per protocol. Sputum examination undertaken in the ward itself was negative for acid fast-Bacilli (AFB). The CXR

This is an open access article distributed under the terms of the Creative Commons Attribution-NonCommercial-ShareAlike 3.0 License, which allows others to remix, tweak, and build upon the work non-commercially, as long as the author is credited and the new creations are licensed under the identical terms.

For reprints contact: reprints@ medknow.com

How to cite this article: Dube P, Saroa R, Palta S. Coinfections in Intensive Care Unit with pulmonary tuberculosis and mucormycosis: A clinical dilemma. Indian J Crit Care Med 2016;20:191-3. 
showed bilateral cavitary lesions in all the zones on the very $1^{\text {st }}$ day of ICU admission. With a high index of suspicion for fungal pneumonia based on the clinical and radiological findings and negative sputum for AFB, antifungal (caspofungin $70 \mathrm{mg}$ intravenous [IV] loading dose followed by $50 \mathrm{mg}$ IV once daily) was started as a part of treatment. HIV and other viral markers were found to be negative on further investigation. However, no clinical improvement was noticed over 5 days, and radiological deterioration was evident by the appearance of new cavitary lesions developing over every $24 \mathrm{~h}$ [Figure 1]. The PF ratio also started deteriorating consistently with the values of 161 on the $3^{\text {rd }}$ day falling to 114.5 on the $9^{\text {th }}$ day with $\mathrm{FiO}_{2}$ going up to 0.9 . With patient condition deteriorating as evidenced by higher ionotropic support and decreasing PF ratio, bronchoalveolar lavage (BAL) was performed, and wastings were sent for AFB staining, pyogenic culture, and fungal culture. AFB staining revealed positive results for AFB. Subsequently, antitubercular treatment was also initiated as per the Revised National Tuberculosis Control Program guidelines for the same. Although the potassium hydroxide wet mount revealed no fungal elements, fungal culture showed growth of Rhizopus species on the $2^{\text {nd }}$ day of inoculation [Figure 2], following which amphotericin B (in the dose of $0.5 \mathrm{mg} / \mathrm{kg}$ IV loading dose followed by $1 \mathrm{mg} / \mathrm{kg}$ once daily) was introduced as per culture sensitivity. On the $11^{\text {th }}$ day, the $\mathrm{PF}$ ratio had further fallen to 71 on $\mathrm{FiO}_{2}$ of 1.0. However, the patient failed to improve and succumbed on the $12^{\text {th }}$ day of admission to ICU.

\section{Discussion}

Opportunistic fungal organisms such as Candida species, Aspergillus species, Mucor species, and Cryptococcus neoformans may be encountered in diabetic patients

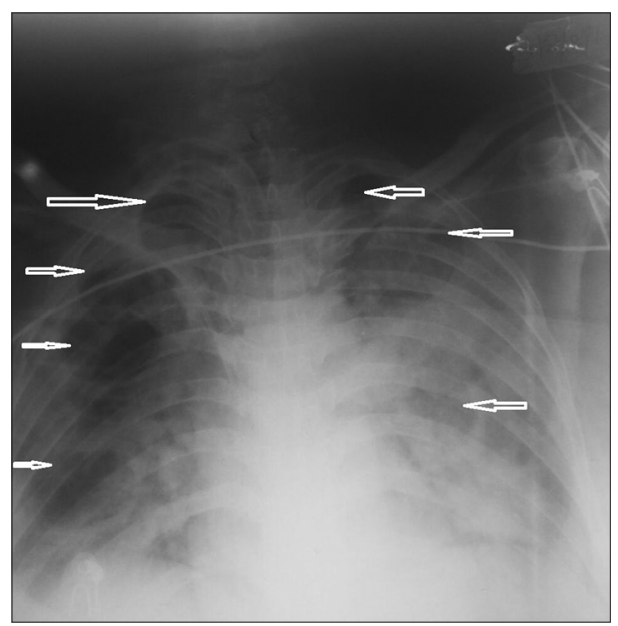

Figure I: Multiple cavitatory lesions on day 5 who are usually immunocompromised by virtue of the disease itself. Diabetes mellitus may predispose to mucormycosis in $36-88 \%$ of patients which is a serious, potentially fatal fungal infection that needs a high degree of suspicion for the diagnosis. ${ }^{[2,3]}$ Patients with uncontrolled hyperglycemia, particularly those with ketoacidosis, are the most susceptible and it may be the first manifestation in patients with undiagnosed diabetes mellitus. ${ }^{[3]}$ The most common presentation is rhinoorbital-cerebral involvement followed by pulmonary infection. ${ }^{[4]}$

Patients with diabetes are prone to develop TB and have high chances of treatment failure. There is also a higher risk of progressing from latent to active TB. A large proportion of people with diabetes further developing TB are not diagnosed, or diagnosed too late, may be due to lack of awareness on the part of the patient to report early for any development of chest symptoms. ${ }^{[5]}$

Coinfection with pulmonary TB and mucormycosis in immunocompromised patients may present a diagnostic dilemma owing to similar clinical presentation and need a high degree of clinical suspicion and early aggressive treatment. ${ }^{[6]}$ Both clinical entities may present with cough, fever, hemoptysis, and/or chest pain. TB being endemic always forms the first diagnosis. In addition, the radiological features of consolidation, cavitation, infiltrates, and effusion are common to both the diseases and, therefore, histopathological examination or culture sensitivity should be undertaken at the earliest to make definitive diagnosis. Clinical and radiological presentations alone are inconclusive toward a particular disease..$^{[7]}$ It is possible that the high relapse cases, treatment failures, resistance, and high mortality

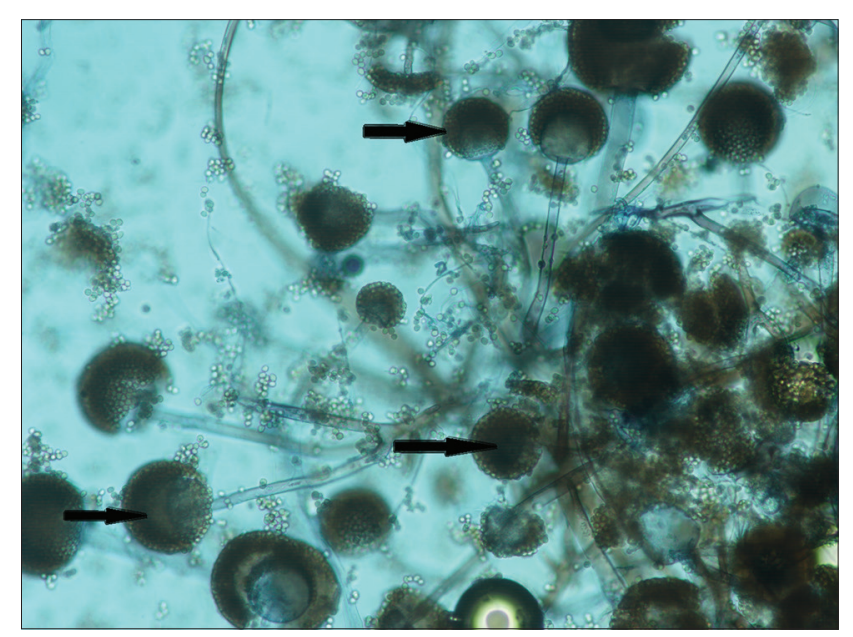

Figure 2: Lactophenol cotton blue mount showing Rhizopus Microsporus species $($ LCB $\times 200)$ 
associated with TB infection are partly attributed to coinfection with opportunistic fungal pathogens and drug-resistant non-TB bacteria. ${ }^{[2]}$

In the present case, an immunocompromised state prevailing as a result of diabetes and TB made the patient prone to acquire coinfection with mucormycosis. Although cavitation was evidenced on the very $1^{\text {st }}$ day of admission to ICU, negative sputum examination in the ward made TB an unlikely diagnosis. An antifungal was started empirically to contain the opportunistic fungal infection. However, conclusive diagnosis was made only after BAL which demonstrated the presence of AFB on smear and ubiquitous filamentous fungus Rhizopus species, of Mucorale order, colonization on culture.

There are isolated reports of coinfection of mucormycosis and TB in immunocompromised patient where the patients were successfully treated. ${ }^{[8,9]}$ In the present case, the unfavorable outcome may be the result of delayed diagnosis which was probably missed for the first 8 days in a ward where primarily the focus was on treating diabetic ketoacidosis. Meanwhile, the hyperglycemic environment that favors immune dysfunction (e.g., damage to the neutrophil function, depression of the antioxidant system, and humoral immunity) flared up coinfection of mucormycosis with latent TB progressing to active TB and followed a fulminant course. ${ }^{[10]}$

\section{Conclusion}

Latent TB and drug-resistant TB in patient with diabetes make them susceptible to opportunistic infections with potentially fatal fungal infections. Simultaneous infection with Mycobacterium tuberculosis and mucormycosis is a rare finding and requires high clinical vigilance. Now-a-days, it is imperative to consider fungal infections as an important differential diagnosis. Early and aggressive treatment targeting the isolated organisms may help reduce the mortality and morbidity. Meanwhile, screening the patients with diabetes for coinfections, especially in nonresolving pneumonias are imperative and should be practiced in a protocolized form in early stages of diabetes.

\section{Financial support and sponsorship}

Nil.

\section{Conflicts of interest}

There are no conflicts of interest.

\section{References}

1. Mwaura EN, Matiru V, Bij C. Mycological finding of sputum samples from pulmonary tuberculosis patients attending TB clinic in Nairobi, Kenya. Virol Mycol 2013;2:119.

2. Brown GD, Denning DW, Gow NA, Levitz SM, Netea MG, White TC. Hidden killers: Human fungal infections. Sci Transl Med 2012;4:165rv13.

3. Greenberg RN, Scott LJ, Vaughn HH, Ribes JA. Zygomycosis (mucormycosis): Emerging clinical importance and new treatments. Curr Opin Infect Dis 2004; $17: 517-25$.

4. Spellberg B, Edwards J, Ibrahim A. Novel perspectives on mucormycosis pathophysiology, presentation, and management. Clin Microbiol Rev 2005; 18:556-69.

5. Collaborative Framework for Care and Control of Tuberculosis and Diabetes. The Stop TB Department: WHO; 2011. Available from: http://www.who.int/diabetes/publications/tb_diabetes2011/en/index. html. [Last accessed on 2015 Jul 15].

6. Garg R, Marak RS, Verma SK, Singh J, Sanjay, Prasad R. Pulmonary mucormycosis mimicking as pulmonary tuberculosis: A case report. Lung India 2008;25:129-31.

7. Machida M, Gomi K. Aspergillus: Molecular Biology and Genomies. UK: Caister Academic Press; 2010. p. 173-98.

8. Sharma SK, Agarwal N, Mukherjee A, Seth T, Mishra P, Xess I, et al. Coexisting pulmonary tuberculosis and mucormycosis in a patient with aplastic anemia post allogenic stem cell transplantation. Mediterr J Hematol Infect Dis 2011;3:e2011036.

9. Aggarwal D, Chander J, Janmeja AK, Katyal R. Pulmonary tuberculosis and mucormycosis co-infection in a diabetic patient. Lung India 2015;32:53-5.

10. Petrikkos G, Drogari-Apiranthitou M. Zygomycosis in immunocompromised non-haematological patients. Mediterr J Hematol Infect Dis 2011;3:e2011012. 